\title{
Hyperdimensional Computing for Noninvasive Brain-Computer Interfaces: Blind and One-Shot Classification of EEG Error-Related Potentials
}

\author{
Abbas Rahimi \\ EECS Department \\ University of California \\ Berkeley \\ Berkeley, CA 94720 \\ abbas@eecs.berkeley.edu
}

\author{
Pentti Kanerva \\ Redwood Center for \\ Theoretical Neuroscience \\ University of California \\ Berkeley \\ Berkeley, CA 94720 \\ pkanerva@berkeley.edu \\ Jan M. Rabaey \\ EECS Department \\ University of California \\ Berkeley \\ Berkeley, CA 94720 \\ jan@eecs.berkeley.edu
}

\author{
José del R. Millán \\ Defitech Foundation Chair in \\ Brain-Machine Interface \\ Ecole Polytechnique Fédérale \\ de Lausanne (EPFL) \\ 1015 Lausanne, Switzerland \\ jose.millan@epfl.ch
}

\begin{abstract}
The mathematical properties of high-dimensional (HD) spaces show remarkable agreement with behaviors controlled by the brain. Computing with HD vectors, referred to as "hypervectors," is a brain-inspired alternative to computing with numbers. HD computing is characterized by generality, scalability, robustness, and fast learning, making it a prime candidate for utilization in application domains such as brain-computer interfaces. We describe the use of HD computing to classify electroencephalography (EEG) errorrelated potentials for noninvasive brain-computer interfaces. Our algorithm encodes neural activity recorded from 64 EEG electrodes to a single temporal-spatial hypervector. This hypervector represents the event of interest and is used for recognition of the subject's intentions. Using the full set of training trials, HD computing achieves on average $5 \%$ higher accuracy compared to a conventional machine learning method on this task $(74.5 \%$ vs. $69.5 \%)$ and offers further advantages: (1) Our algorithm learns fast by using $34 \%$ of training trials while surpassing the conventional method with an average accuracy of $70.5 \%$. (2) Conventional method requires prior domain expert knowledge to carefully select a subset of electrodes for a subsequent preprocessor and classifier, whereas our algorithm blindly uses all 64 electrodes, tolerates noises in data, and the resulting hypervector is intrinsically clustered into HD space; in addition, most preprocessing of the electrode signal can be eliminated while maintaining an average accuracy of $71.7 \%$.
\end{abstract}

Permission to make digital or hard copies of all or part of this work for personal or classroom use is granted without fee provided that copies are not made or distributed for profit or commercial advantage and that copies bear this notice and the full citation on the first page. To copy otherwise, to republish, to post on servers or to redistribute to lists, requires prior specific permission and/or a fee. BICT 2017, March 15-16, Hoboken, United States ISBN 978-1-63190-148-5

DOI: 10.4108/eai.22-3-2017.152397

Copyright $\odot 2017$ EAI

\section{CCS Concepts}

-Computing methodologies $\rightarrow$ Bio-inspired approaches; $\bullet$ Theory of computation $\rightarrow$ Probabilistic computation; Unsupervised learning and clustering;

\section{Keywords}

Brain-inspired computing, hyperdimensional computing, EEG, error-related potentials, brain-computer interfaces, machine learning

\section{INTRODUCTION}

Over the past six decades, the semiconductor industry has been immensely successful thanks to the set of welldefined abstraction layers, starting from robust switching devices that support a deterministic Boolean algebra and going up to a scalable and stored program architecture, which is Turing complete, and hence capable of tackling (almost) any computational challenge. Unfortunately this abstraction chain is being challenged as device scaling continues to nanometer dimensions and also by exciting new applications that must support a myriad inputs such as brain-computer interfaces [1]. A brain-computer interface is a device that enables communication and control without movement. For these applications, cognitive functions such as classification, recognition, synthesis, decision-making and learning are of crucial importance for fast and efficient informationextraction. This is in sharp contrast to the past when the central objective of computing was to perform calculations on numbers and produce results with extreme numerical accuracy. It is therefore worth exploring alternative computational models for emerging applications by abandoning the deterministic requirement.

Brain-inspired information processing architectures provide significant increase in energy efficiency, asymptotically approaching the efficiency of brain computation, while aligning well with the variability of nanoscale devices $[9,19$, 
16, 21]. The mathematical properties of high-dimensional spaces correlate strongly with behaviors controlled by the brain $[15,22,12,14]$. We focus on a model of computing with high-dimensional (HD) vectors-hereafter HD computing - where the dimensionality is in the thousands (e.g., 10,000 dimensions). HD computing is also referred to as "hyperdimensional computing" [12] on account of the very high dimensionality. In this formalism, information is represented in HD vectors referred to as "hypervectors". HD computing explores the emulation of cognition by computing with hypervectors as an alternative to computing with bits and numbers. Hypervectors are holographic and (pseudo)random vectors with i.i.d. components. It means that every piece of information contained in the hypervector is distributed equally over all the components. Such hypervectors can then be mathematically manipulated to not only classify but also to bind, associate, and perform other types of cognitive operations in a straightforward manner [13]. In addition, these mathematical operations also ensure that the resulting hypervector is unique and thus learning can take place in one-shot.

Key properties of HD computing include: (1) HD computing paradigm is universal and complete. (2) By its very nature, HD computing overcomes large variability and uncertainty in both data and implementation platform to perform robust decision making and classification. (3) In contrast to other neuro-inspired approaches, in which learning is separate from subsequent execution, learning in HD computing shares its constructs with execution, is relatively fast, and does not rely on biologically unlikely algorithms such as back-propagation. (4) HD computing is memory-centric by manipulating and comparing large patterns, within the memory using ubiquitous parallel operations. Such generality, robustness against data uncertainty, and one-shot learning make HD computing a prime candidate for utilization in application domains such as brain-computer interfaces and wearable cyberbiological systems.

HD computing has been used for text analytics solely from a stream of input letters. More specifically, HD computing can identify the language of unknown sentences from $21 \mathrm{Eu}-$ ropean languages $[10,21]$, and classify Reuters news articles to eight topics [18] with very high accuracy. It has also been adapted to wearable biosignal processing characterized by a set of parallel and analog streaming inputs. Very simple vector-space operations are used to classify hand gestures from four electromyography (EMG) electrodes [20].

In this paper, we further extend the application domain of HD computing to noninvasive brain-computer interfaces. We develop an encoding algorithm and a classifier for recognition of the subject's intentions from error-related electroencephalography (EEG) potentials. Our algorithm encodes neural activity that is recorded simultaneously by 64 EEG electrodes, to a single temporal-spatial hypervector representing the intention of a subject. Our proposed HD classifier surpasses the state-of-the-art method [4] - referred to as baseline in this paper-for classifying EEG errorrelated potentials in the following aspects: (1) With an equivalent setup, HD classifier achieves an average classification accuracy of $74.5 \%, 5 \%$ higher than the baseline. (2) HD classifier learns $\approx 3 \times$ faster by using only $34 \%$ of training trials while maintaining average accuracy of $70.5 \%$ which is higher than the baseline using the full set of training trials. (3) HD classifier blindly uses all 64 electrodes without requiring any domain expert knowledge for the classification task, and the resulting hypervector is intrinsically clustered into HD space; in contrast, the authors in [4] carefully chose one or two electrodes, depending upon the subject, to be used for the baseline classifier. Moreover, (4) most preprocessing of the electrode signal can be eliminated in our classifier. These blind operation with all electrodes and less preprocessing result in minimal loss of accuracy (from $74.5 \%$ to $71.7 \%$ ). MATLAB code for our encoding algorithm and classifier is open access ${ }^{1}$.

This paper is organized as follows. In Section 2, we describe EEG error-related potentials and its usage in noninvasive brain-computer interfaces followed by its baseline preprocessing and classification. In Section 3, we introduce HD computing and discuss how its operations can be used to form an HD classifier. In Section 4, we present our algorithm for encoding and classifying EEG error-related potentials. In Section 5, we provide experimental results followed by discussion in Section 6. Section 7 concludes this paper.

\section{NONINVASIVE BRAIN-COMPUTER INTERFACES}

Noninvasive brain-computer interfaces and neuroprostheses aim to provide a communication and control channel based on the recognition of the subject's intentions from spatiotemporal neural activity typically recorded by EEG electrodes. What makes it particularly challenging, however, is its susceptibility to errors in the recognition of human intentions.

\subsection{EEG Error-Related Potentials}

As an alternative interaction approach, the user can monitor the performance of an autonomous agent endowed with learning capabilities, and the erroneous behavior of the agent can be recognized directly from the analysis of the user's brain signals, i.e., EEG error-related potentials (ERP). In the frame of brain-computer interaction, Ferrez and Millán have described an ERP elicited by errors in the recognition of the user's intention when operating a brain-computer interface $[7,6]$. In their experimental protocol, the human subject tries to move a cursor towards a target location either using a keyboard [7] or mental commands [6]. Next, they have observed that similar error-related signals are generated when a human user monitors the performance of an external agent upon which the user has no control [4]. In this approach, the user does not provide any commands, but only monitors the agent's performance. Efficient and fast learning methods of encoding these ERPs for accurate classifying user's intentions with regard to the agent further motivates its application for noninvasive brain-computer interfaces.

\subsection{Dataset for Error-Related Potentials}

Here, we first describe a publicly available dataset [2] for ERPs, and then outline the baseline method [4] that is used for processing of these potentials. Six subjects are seated in front of a computer screen where a moving cursor is displayed. A colored square at either the left or right of the cursor indicates a target location. At each trial the cursor moves horizontally depending on the location of the target. During the experiment, the user has no control over the

${ }^{1}$ https://github.com/abbas-rahimi/HDC-EEG-ERP 
cursor's movement and is asked only to monitor the performance of the agent, knowing that the goal is to reach the target. To study signals generated by erroneous actions, at each trial, there is a probability of $\approx 0.20$ for the cursor to move in the wrong direction (i.e., opposite to the target location). A trial is labeled as "correct" if the curser is moved toward the target; otherwise it is labeled as "error", e.g., when the target is located in the left and the cursor is moved to the right.

Trials have an approximate duration of $2000 \mathrm{~ms}$. There are two recording sessions, the first one is used for training, and the second one is used for testing. Each experimental session consists of 10 blocks of 3 min each $(\approx 64$ trials per block). Full details of the experimental protocol are provided in [4]. In the following, we explain their methods for EEG signal acquisition, preprocessing, and classification. We refer them as the baseline for comparing with our HD computing method.

\subsubsection{Baseline Preprocessing and Classification}

EEG potentials were recorded at a sampling rate of 512 $\mathrm{Hz}$ using 64 electrodes according to the standard 10/20 international system. For preprocessing, data was spatially filtered using common average reference (CAR) [17]. By applying the CAR filter to an electrode, the average signal level of the entire electrode array is subtracted from that of the electrode of interest. If the entire head is covered by equally spaced electrodes and the potential on the head is generated by point sources, the CAR results in a spatial voltage distribution with a mean of zero [3]. We show in Section 5.2 that this spatial filter preprocessing can be eliminated with negligible effect on the classification accuracy thanks to the $\mathrm{HD}$ operations that can work on raw data. Then, a $1-10 \mathrm{~Hz}$ band-pass filter (BPF) was applied to remove the unwanted frequency components. For every subject, a time window corresponding to erroneous and correct cursor movements was extracted for further analysis and classification (listed in the third column of Table 1).

A Gaussian classifier was used for recognition of a single trial, as described in [7]. This statistical classifier estimates the posterior probability of a given trial corresponding to one of the two classes: "error," and "correct". $\mathrm{FCz}$ and $\mathrm{Cz}$ electrodes were used as the inputs to the classifier following their earlier studies for electrode selection process [5]. The same learning rates and number of prototypes were used in all cases. Classifier parameters are then tuned using a stochastic gradient descent on the mean square error [7]. To tune the classification performance, the choice of electrodes ( $\mathrm{FCz}, \mathrm{Cz}$, or both) and time windows were selected independently per subject [4] (see Table 1).

Our aim is to develop an efficient and fast learning method based on HD computing that replaces the aforementioned preprocessing and classification enabling blindly operating with all electrodes, and with raw data. We provide backgrounds about HD computing in Section 3, and then present details of our method in Section 4.

\section{HD COMPUTING}

The brain's circuits are massive in terms of numbers of neurons and synapses, suggesting that large circuits are fundamental to the brain's computing. HD computing $[12,14]$ explores this idea by looking at computing with ultra-wide words - that is, with hypervectors. There exist a huge num- ber of different, nearly orthogonal hypervectors with the dimensionality in the thousands (e.g., $D=10,000$ ) [11]. This lets us combine two such hypervectors into a new hypervector using well-defined vector-space operations, while keeping the information of the two with high probability.

Hypervectors are made using random indexing [15, 22] with operations akin to multiplication, addition, and permutation that form an algebra over the vector space (e.g., a field). Random indexing represents information by projecting data onto hypervectors. It is incremental, scalable, and computes hypervectors in a single pass over the input data. Random indexing with a bipolar dense code generates hypervectors that are initially taken from a 10,000-dimensional space and have equally probable randomly placed +1 s and -1 s, i.e., $\{-1,+1\}^{10,000}$

\subsection{Item Memory}

Item memory (iM) is a symbol table or dictionary of all the hypervectors defined in the system. In a typical language application, the 26 letters of the alphabet and the space are the initial items, and they are assigned hypervectors at random (with i.i.d. components). They stay fixed throughout the computation, and they serve as seeds from which further representations are made. HD computing has been used for identifying the source language of text samples from a sequence of $n$ consecutive letters ( $n$-grams) [10, 21]. For example, letter trigrams of a text sample were encoded into a hypervector by random indexing and vector-space operations to represent a language. In the same vein, pentagrams of letters have been used for classifying news articles [18].

Text and language applications are well-matched to the HD computing framework because the input data already comes in the form of symbolic primitives (letters, or words), which can be readily mapped to hypervectors. On the other hand, biosignal processing applications often operate on analog time series with multiple sensory inputs demanding a different mapping scheme to hypervectors. Accordingly, we have extended the notion of iM to a continuous item memory (CiM) that maps an analog input after a discretization step [20]. CiM utilizes a method of mapping quantities and dates "continuously" to hypervectors [23]. In this continuous vector space, orthogonal endpoint hypervectors are generated for the minimum and maximum levels in the range. Hypervectors for intermediate levels are then generated by linear interpolation between these endpoints so that the similarity of vectors corresponds to the closeness of levels.

For example, if an analog signal is discretized into $m$ levels, we choose a random hypervector for the minimum level and randomly flip $D / 2 /(m-1)$ of its bits for each successively higher level (once flipped, a bit will not be flipped

Table 1: Classifier parameters: selected electrodes and time windows used in Gaussian classifier [4]; and $n$-gram sizes for our HD classifier.

\begin{tabular}{llll}
\hline Subjects & Electrodes & Time window $(\mathrm{ms})$ & $n$-gram \\
\hline S1 & $\mathrm{FCz}, \mathrm{Cz}$ & $200-450$ & 16 \\
\hline $\mathrm{S} 2$ & $\mathrm{Cz}$ & $150-600$ & 29 \\
\hline $\mathrm{S} 3$ & $\mathrm{FCz}, \mathrm{Cz}$ & $200-450$ & 16 \\
\hline $\mathrm{S} 4$ & $\mathrm{FCz}$ & $0-600$ & 19 \\
\hline S5 & $\mathrm{FCz}, \mathrm{Cz}$ & $150-600$ & 29 \\
\hline $\mathrm{S} 6$ & $\mathrm{FCz}, \mathrm{Cz}$ & $150-600$ & 29 \\
\hline
\end{tabular}


back). The vectors for the minimum and the maximum levels will then be $D / 2$ bits apart or orthogonal to each other.

\subsection{MAP Operations}

The seed hypervectors that are stored in iM and CiM can be further combined using the following well-defined set of arithmetic operations. We consider a variant of the multiplication, addition, and permutation (MAP) coding described in [8] to define the hyperdimensional vector space. The MAP operations on the hypervectors are defined as follows. Pointwise multiplication of two hypervectors $A$ and $B$ is denoted by $A * B$, and point-wise addition is denoted by $A+B$. Multiplication takes two vectors and yields a third, $A * B$, that is dissimilar (orthogonal) to the two and is suited for variable binding; and addition takes several vectors and yields their mean vector $[A+B+\ldots+X]$ that is maximally similar to them and is suited for representing sets. In the following, we describe the use of these two operations to holistically encode a data record composed of various fields [13].

A data record consists of a set of fields (variables/attributes) and their values (fillers); for example, the variables $x, y, z$ with values $a, b, c$, respectively. The holistic encoding is done as follows. The field-value pair $x=a$ is encoded by the hypervector $X * A$ that binds the corresponding hypervectors, and the entire record is encoded by the hypervector $R=[(X * A)+(Y * B)+(Z * C)]$ which includes both the variables and the values, and each of them spans the entire 10,000-bit hypervector.

Finally, the third operation is a permutation, $\rho$, that rotates the hypervector coordinates. It is implemented as a cyclic right-shift by one position. The permutation operation generates a dissimilar pseudo-orthogonal hypervector that is good for encoding sequences. In geometry sense, the permutation rotates the hypervector in the space. For example, the sequence trigram of a-b-c, is stored as the hypervector $\rho(\rho A * B) * C=\rho \rho A * \rho B * C$. This efficiently distinguishes the sequence a-b-c from a-c-b, since a rotated hypervector is uncorrelated to all the other hypervectors.

\subsection{Associative Memory}

Hypervectors can be stored in an associative memory to be compared for similarity using a distance metric over the vector space. We use cosine similarity as the distance metric between two hypervectors by measuring the cosine of the angle between them using a dot product. It is defined as $\cos (A, B)=\left|A^{\prime} * B^{\prime}\right|$, where $A^{\prime}$ and $B^{\prime}$ are the lengthnormalized vectors of $A$ and $B$, respectively, and $|C|$ denotes the sum of the elements in $C$. It is thus a measure of orientation and not magnitude: two hypervectors with the same orientation have a cosine similarity of 1 , two orthogonal hypervectors have a similarity of 0 , and two hypervectors diametrically opposed have a similarity of -1 .

\section{HD COMPUTING FOR EEG ERP}

In this section, we describe how HD computing can be used to encode ERPs into hypervectors. Our proposed encoder first captures a sequence of electrical activities of an electrode into a temporal hypervector (Section 4.1) and then encodes the information across all the electrodes at a moment into a temporal-spatial hypervector (Section 4.2) for the HD classifier (Section 4.3). Figure 1 shows the structure of proposed temporal-spatial encoder.

\subsection{Temporal Encoder for One Electrode}

There are 64 EEG electrodes with unique names, and each electrode produces an analog signal with an amplitude. We draw an analogy from [13] to generate a holistic vector representing information about a given electrode by using a field-value pair. Hence, we decouple the name and the signal level of an electrode. The electrode name corresponds to a field of a traditional data record, and its signal level corresponds to the value for the field. Since the name of every electrode is a unique string, it forms a field that can be easily mapped to a hypervector $(N)$ using an iM with 64 entries. The iM represents the 64 basic fields by assigning a unique orthogonal hypervector to every field: $N_{1} \perp N_{2} \ldots \perp N_{64}$.

$$
N_{i}=\mathrm{iM} \text { (name of } i \text { th electrode) }
$$

Although the names of electrodes can be readily mapped to hypervectors, mapping their signal levels requires a quantization step. Here, the signal level can be the raw analog data or the preprocessed data as described in Section 2.2.1. The signal levels of each electrode are scaled linearly from 0 to 100 , and quantized into 100 discrete levels. This quantized signal level is mapped to a hypervector $(L)$ using a CiM with 100 entries. In CiM, the hypervector that is assigned to the minimum signal level is orthogonal to the hypervector representing the maximum signal level: $\mathrm{CiM}(0) \perp \mathrm{CiM}(99)$.

An event of ERP is not a single signal sample but rather a sequence of samples spanned over a time window forming a temporal component. Hence, we should design a temporal encoder to capture all the signal levels generated during the entire event of an ERP. We can encode a sequence of symbols by using the permutation operation, $\rho$. As shown in Section 3.2, permutation has been used to encode a sequence of $n$ letters to form an $n$-gram hypervector. By analogy, for example, a sequence of three signal levels of electrode $i$ that are generated at time stamps of 1,2 , and 3 is encoded as follows: the first hypervector $L_{i 1}$ is rotated twice $\rho^{2} L_{i 1}$, the second hypervector is rotated once $\rho^{1} L_{i 2}$, and finally there is no rotation for the last hypervector $L_{i 3}$. These three hypervectors are then combined with point-wise multiplication into a trigram hypervector, $G_{i}=\rho^{2} L_{i 1} * \rho^{1} L_{i 2} * L_{i 3}$. For $n$-grams at large this becomes:

$L_{i t}=$ CiM (signal level of $i$ th electrode at time stamp $t$ )

$$
G_{i}=\prod_{t=1}^{n} \rho^{n-t} L_{i t}
$$

With this temporal encoding algorithm, one important step is to determine the proper size of an $n$-gram to be able to capture the entire event of an ERP for the subjects. In this regard, we measured the number of samples available in an event of ERP. Table 1 shows the time windows in which the events of ERP are observed during the trials of $2000 \mathrm{~ms}$ for each subject. As shown, this time window can be as large as $600 \mathrm{~ms}$ (for S4) containing 308 samples with sampling rate of $512 \mathrm{~Hz}$. Computing an $n$-gram of this size is inefficient; earlier, we overcame such issue for the EMG signals by highly downsampling the electrode such that the hand gestures can be fit into $n$-grams where $n \in[3,5][20]$. Similarly, the electrical activity on the EEG electrodes was downsampled to $64 \mathrm{~Hz}$ [4]. Consequently, we partition the time widow of ERP to a set of nonoverlapping slices of equal length. The length of every slice is 8 samples as the ratio of 


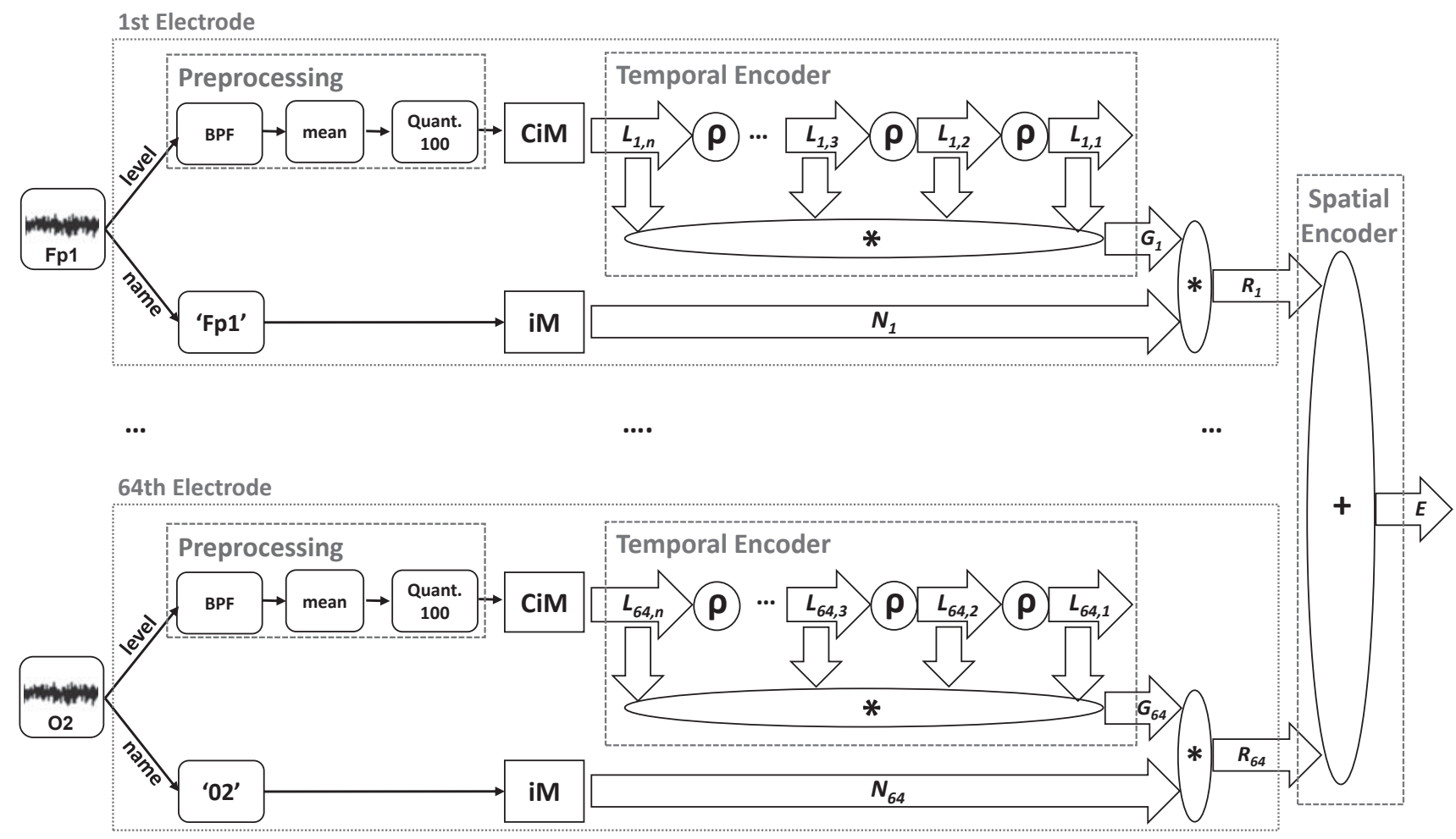

Figure 1: Temporal-spatial encoder for EEG ERPs: Temporal encoder rotates the signal level to capture its history that produces a temporal $n$-gram $\left(G_{i}\right)$ to be bound with the electrode name $\left(N_{i}\right)$ for constructing a record $\left(R_{i}\right)$; Spatial encoder adds these records across 64 electrodes to produce a temporal-spatial hypervector $(E)$ representing the entire event of ERPs.

sampling rate $(512 \mathrm{~Hz})$ to the downsampling rate $(64 \mathrm{~Hz})$. With 8 samples in a slice, the number of slices in the window is proportional to the duration of the window, and varies from 16 slices to 29 slices $^{2}$. Then, we take the mean value of samples in the slice, apply the scaling and quantization step and use it as the quantized signal level for CiM. Therefore, the event of ERP can be represented by an $n$-gram where $n \in$ $[16,29]$. The last column in Table 1 lists the exact size of $n$ gram used for each subject. Please note that the $n$-gram size is the only parameter of our encoder that we naturally set it per subject solely based on the duration of ERP event. This is in sharp contrast with other learning methods as we do not rely on inefficient or biologically implausible algorithm for parameter tuning and optimizations.

Our temporal encoder accepts the sequence of $n$ quantized signal levels from the $i$ th electrode and computes the $n$-gram hypervector $G_{i} . G_{i}$ represents the temporal activities of the signal levels of the $i$ th electrode.

\subsection{Temporal-Spatial Encoder: Adding Tem- poral Hypervectors of 64 Electrodes}

The 64 temporal hypervectors $G_{i}$ for the electrodes are encoded into a single temporal-spatial hypervector $E$ for the event in a manner analogous to the holistic record of [13]. The electrode name is treated as a field, and its signal is treated as a value for the field. The channel names $N_{1}, N_{2}, \ldots, N_{64}$ are hypervectors that reside in the item

${ }^{2}$ For S4, we double the length of slices (16 samples in each) that results in 19 slices to cover the window instead of 38 . memory, iM. They are random, independent, and approximately orthogonal to one another. The signal $G_{i}$ is bound to its channel name $N_{i}$ with point-wise multiplication and is represented by the hypervector

$$
R_{i}=G_{i} * N_{i}
$$

and the entire event is represented by a hypervector $E$ that is the sum of 64 hypervectors for the field-value pairs:

$$
E=\sum_{i=1}^{64} R_{i}
$$

The structure of the encoder is shown in Figure 1.

\subsubsection{Class Prototypes}

The classifier construction is purely based on native operations of HD computing without the involvement of any optimization procedure. The approach is based on the notion of a class prototype. The class prototype is a hypervector representing the entire class. The number of class prototypes equals the number of classes in ERP. Hence, we generate two hypervectors $C$ and $W$ as the class prototypes for ERP: $C$ represents the "correct" class and $W$ represents the "error" (wrong) class. The hypervector $C$ is computed by adding all the hypervectors produced by the temporalspatial encoder from the "correct" events of ERP. Hence, the addition operation bundles $E_{j}$ observed in training trial $j$ to a single hypervector $C$ as follows:

$$
C+=E_{j} \mid \cos \left(C, E_{j}\right)<0.5
$$


Before adding a new event $E_{j}$ to $C$, we check whether this event is already in $C$. This checking forms a conditional addition that adds $E_{j}$ to $C$ when $\cos \left(C, E_{j}\right)<0.5$. If $C$ has a high cosine similarity $(\geq 0.5)$ with $E_{j}$, it means that the event is already in $C$, hence there is no need to add the redundant event. Similarly, the hypervector $W$ is computed for the "error" events.

\subsection{HD Classification}

After training, $C$ and $W$ hypervectors are stored in the associative memory as the learned patterns of ERP. The same encoding algorithm is used for both training and testing (i.e., classification). When testing, we call the output of the temporal-spatial encoder a query hypervector $(Q)$ since its label is unknown. The query hypervector is then sent to the associative memory to identify its source class. Determining the class of an unknown ERP event is done by comparing its $Q$ hypervector to all learned hypervectors (i.e., $C$ and $W$ ) using the cosine similarity. Finally, the associative memory selects the highest similarity among the two measures and returns its associated label as the class that the $Q$ hypervector has been generated from.

\section{EXPERIMENTAL RESULTS}

In this section, we present experimental results and sensitivity analyses for classification accuracy of our proposed HD method. We compare it with the baseline method presented in [4] using their dataset available at [2]; session \#1 is used for training, and session \#2 is used for testing the accuracy. The classification accuracy throughout this paper is measured as macroaveraging that computes a simple average over classes. The macroaveraging gives equal weight to each class, whereas microaveraging gives equal weight to each per-event classification decision. The macroaveraging is a better measure of effectiveness with small classes (the size of "correct" class is $\approx 4 \times$ of the "wrong" class). Our HD encoding algorithm and classifier are developed in MATLAB.

\subsection{Fast and One-Shot Learning}

Here, we assess how fast the HD training can be done while maintaining high classification accuracy. As described in Section 2.2, the training session is composed of 10 blocks of recording that are shown by vertical dashed lines in Figure 2. These consecutive blocks provide a total number of $\approx 640$ trials for training per subject. We have observed that only some of these training trials can produce a nonredundant temporal-spatial hypervector for addition to the class prototype (i.e., meeting Equation 6). For instance, as shown in Figure 2, the training session contains 191 and 348 non-redundant trials for S3 and S5, respectively. Hence, during the training session, every time that we encounter a new non-redundant ERP event, we update the associative memory and measure the classification accuracy for the entire test set.

Figure 2(a) shows the classification accuracy of both classes for S3. For the very first trials the associative memory is almost empty, but as we encounter new trials it will be lightly populated leading to an increase in the microaveraged accuracy. Training with only the first five non-redundant trials $(2.6 \%$ of the total), the accuracy of HD classifier reaches to $79.3 \%$ which is higher than the baseline accuracy $(75.9 \%)$ using all training trials. Similarly but with a slower learning rate, the HD classifier achieves the accuracy of $66.3 \%$ for
S5 by using 183 non-redundant trials (53\% of the total) as shown in Figure 2(b).

We repeat the aforementioned experiment for all subjects and the results are summarized in Figure 3. We target the classification accuracy of the baseline that is achieved by using all available trials in the training session, one or two electrode(s) (c.f. Table 1), and with the CAR preprocessing technique. We provide the same conditions for the HD classifier $^{3}$, but with fewer training trials, to assess how fast it can reach to the target accuracy. As shown, the HD classifier is able to learn faster: it uses only $0.3 \%$ of the non-redundant training trials for S6, and up to $96 \%$ for S1. On average, the HD classifier reaches the target classification accuracy of $70.5 \%$ when trained with only $34 \%$ of non-redundant training trials. This translates directly to $\approx 3 \times$ faster learning.

\subsection{Blindly Using all Electrodes without Pre- processing}

Figure 4 compares the classification accuracy of the baseline method with two instances of our HD classifier. The first one has a setup equivalent of the baseline as aforesaid: uses one or two electrode(s) based up on the subjects, applies the CAR preprocessing filter on every electrode before the BPF step, and uses the entire of training trials. As shown in Figure 4, this instance of HD classifier surpasses the baseline accuracy across the six subjects. The HD classifier exhibits $67.7 \%-82.7 \%$ classification accuracy and on average $74.5 \%$ which is $5 \%$ higher than the baseline with the same conditions.

Next, we want to assess the ability of HD classifier to blindly operate with raw data from all the 64 EEG electrodes without requiring the prior domain expert knowledge to carefully select a subset of the electrodes for a preprocessor. Hence, the second instance of our HD classifier operates with the 64 electrodes and without the CAR preprocessing filter. It is illustrated in Figure 1 where the temporal-spatial encoder accepts the inputs from the 64 electrodes; every electrode signal is immediately passed through a BPF, and its mean is computed over 8 samples followed by the scaling and quantization step before mapping to the HD space by a CiM. As shown, there is no CAR filter in the chain of data. Note that the simple BPF cannot be removed since the ERPs are in the frequency range of $1-10 \mathrm{~Hz}$.

Despite using the 64 electrodes and no CAR filtering, the HD classifier maintains almost the same range of classification accuracy (i.e., $62.3 \%-79.1 \%$ ) across the six subjects as shown in Figure 4. This HD classifier shows on average $2.2 \%$ higher classification accuracy compared to the baseline. Note that this has been accomplished while this classifier blindly uses all 64 electrodes in the encoder regardless of the subjects, while for the baseline authors carefully selected a subset of electrodes per individual subject that can provide meaningful information for the classifier (listed in the first column of Table 1). This also confirms the amenability of HD computing to operate with raw data. In HD computing, the input data is naturally clustered in HD space, and the noise generated by meaningless electrodes tends to cancel out. This desirable property makes it possible to apply HD computing for clustering data with little or no prior knowledge about the nature of data.

${ }^{3}$ Equation 5 for the spatial encoder is limited to one or two
electrode(s) and the CAR filter is applied before the BPF. 


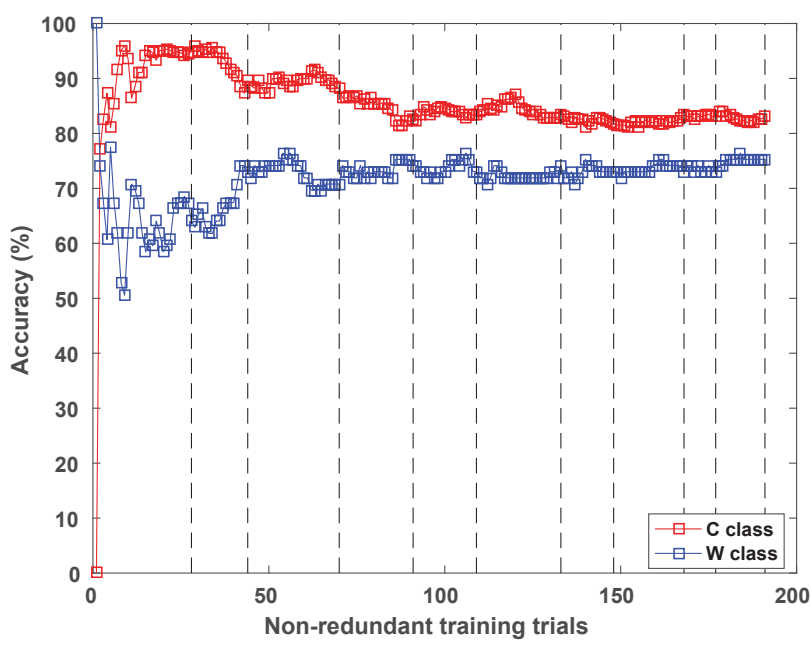

(a) S3

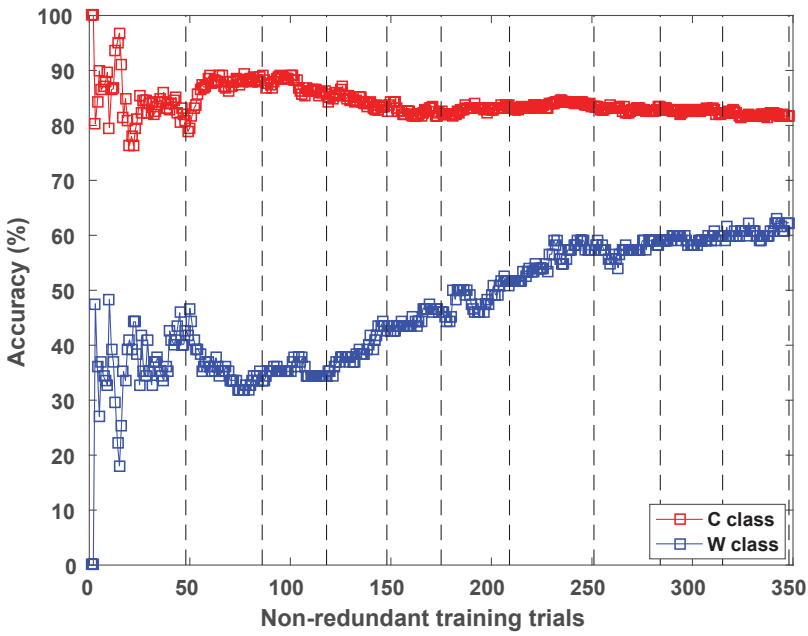

(b) S5

Figure 2: Classification accuracy of both classes when we increase the size of non-redundant training trials for two subjects: (a) S3 and (b) S5. Dashed lines separate the 10 blocks of training session.

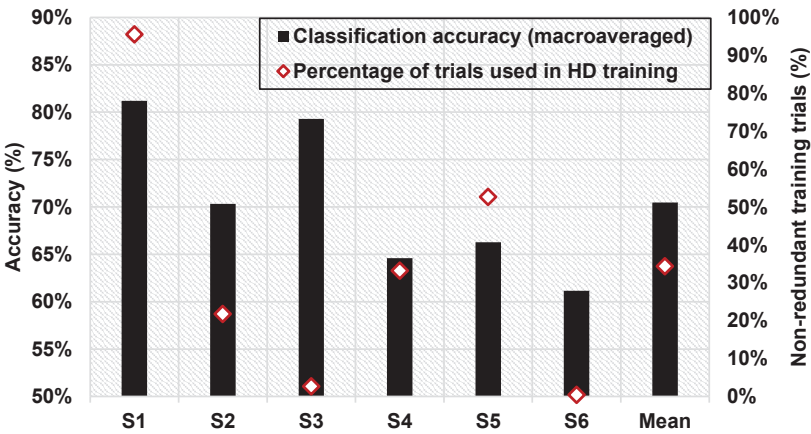

Figure 3: The percentage of non-redundant training trials that are used to train the HD classier, with resulting accuracy, instead of using all trials.

\section{DISCUSSION}

We have earlier used HD computing to encode biosignals from EMG electrodes for hand gesture recognition [20]. We used a spatial-temporal encoder with small $n$-gram sizes to deal with EMG recoding from four electrodes. However, encoding EEG data requires larger $n$-gram sizes which brings up the following issue. In the spatial encoder, the point-wise addition of bipolar hypervectors produces 0s and the pointwise multiplication in the temporal encoder makes them contagious. After a certain size of $n$, this results in generating an $n$-gram hypervector where all elements are 0 .

We can start with bipolar hypervectors, meaning that a sum hypervector can have 0s. We can leave the 0s alone, except that when we multiply two hypervectors, we check for 0 s and turn them into $\mathrm{a}+1$ or $\mathrm{a}-1$ at random. This introduces noise but keeps the hypervectors from eventually turning into all $0 \mathrm{~s}$. We will be able to make vectors for $n$ grams of any length, but the amount of noise will increase with the length of the $n$-gram. The noise grows as follows. If the noise probabilities of two independent bipolar hypervectors are $p_{1}$ and $p_{2}$, the noise probability of their product is

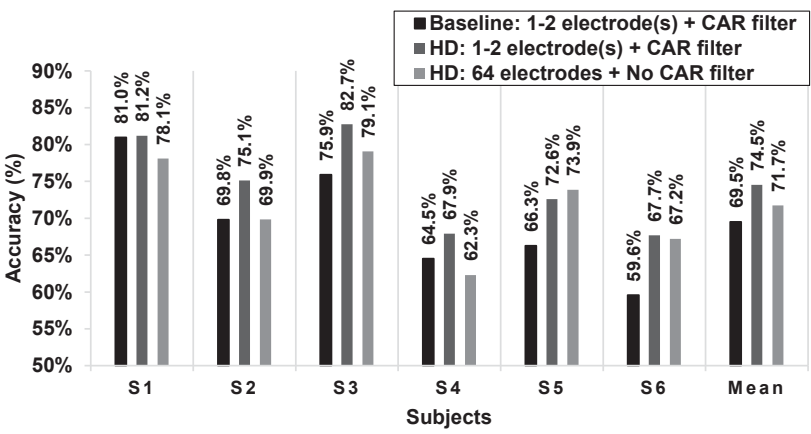

Figure 4: Comparison of microaveraged accuracy of the baseline [4] classifier with two instance of our HD classifier: (1) Using 1 or 2 electrode(s) and CAR preprocessing as in the baseline; (2) Using 64 electrodes without CAR preprocessing.

$p_{1}+p_{2}-2 p_{1} p_{2}$. In contrast, if we let $0 \mathrm{~s}$ be, they grow faster, at the rate of $p_{1}+p_{2}\left(1-p_{1}\right)=p_{1}+p_{2}-p_{1} p_{2}$. Therefore, in this paper, we chose to change the order of encoders: first doing the temporal encoding of each electrode that produces a bipolar hypervector, and then doing the spatial addition.

\section{CONCLUSION}

This paper presents an application of HD computing to the classification of error-related potentials from EEG recordings. Very simple vector-space operations are used to encode analog input signals from 64 electrodes for classification. The proposed HD classifier requires neither prior knowledge about selecting the electrodes nor extra preprocessing steps. Our HD algorithm blindly encodes the electrical activity of error-related potentials into a temporalspatial hypervector representing a binary class of the subject's intentions. The classification accuracy of our HD classifier for EEG error-related potentials is comparable to a 
classifier crafted by a skilled professional. Our HD classifier also achieves it with fewer training data.

\section{ACKNOWLEDGMENT}

This work was supported by Systems on Nanoscale Information fabriCs (SONIC), one of the six SRC STARnet Centers, sponsored by MARCO and DARPA, and by Intel Strategic Research Alliance (ISRA) program on Neuromorphic Architectures for Mainstream Computing.

\section{REFERENCES}

[1] BNCI Horizon 2020. http://bnci-horizon-2020.eu/.

[2] Monitoring error-related potentials. http://bnci-horizon-2020.eu/database/data-sets.

[3] O. Bertrand, F. Perrin, and J. Pernier. A theoretical justification of the average reference in topographic evoked potential studies. Electroencephalography and Clinical Neurophysiology/Evoked Potentials Section, 62(6):462 - 464, 1985.

[4] R. Chavarriaga and J. d. R. Millán. Learning from EEG error-related potentials in noninvasive brain-computer interfaces. IEEE Transactions on Neural Systems and Rehabilitation Engineering, 18(4):381-388, Aug 2010.

[5] P. Ferrez and J. d. R. Millán. Error-related EEG potentials in brain-computer interfaces. $\mathrm{PhD}$ thesis, STI, Lausanne, 2007.

[6] P. W. Ferrez and J. del R. Millán. Error-related EEG potentials generated during simulated brain-computer interaction. IEEE Transactions on Biomedical Engineering, 55(3):923-929, March 2008.

[7] P. W. Ferrez and J. d. R. Millán. You are wrong! - automatic detection of interaction errors from brain waves. In Proceedings of the 19th International Joint Conference on Artificial Intelligence, 2005.

[8] R. W. Gayler. Multiplicative binding, representation operators and analogy. Advances in analogy research, New Bulgarian University, 1998.

[9] J. Hsu. IBM's new brain [news]. IEEE Spectrum, 51(10):17-19, October 2014.

[10] A. Joshi, J. Halseth, and P. Kanerva. Language geometry using random indexing. Quantum Interaction 2016 Conference Proceedings, QI 2016, July 20-22, San Francisco.

[11] P. Kanerva. Sparse Distributed Memory. MIT Press, Cambridge, MA, USA, 1988.

[12] P. Kanerva. Hyperdimensional computing: An introduction to computing in distributed representation with high-dimensional random vectors. Cognitive Computation, 1(2):139-159, 2009.

[13] P. Kanerva. What we mean when we say "what's the dollar of Mexico?": Prototypes and mapping in concept space. In AAAI Fall Symposium: Quantum Informatics for Cognitive, Social, and Semantic Processes, pages 2-6, 2010.

[14] P. Kanerva. Computing with 10,000-bitwords. In Proc. 52nd Annual Allerton Conference on Communication, Control, and Computing, 2014.

[15] P. Kanerva, J. Kristoferson, and A. Holst. Random indexing of text samples for latent semantic analysis.
In Proceedings of the 22nd Annual Conference of the Cognitive Science Society, pages 1036. Erlbaum, 2000.

[16] H. Li et al. Hyperdimensional computing with 3D VRRAM in-memory kernels: Device-architecture co-design for energy-efficient, error-resilient language recognition. IEEE International Electron Devices Meeting, 2016.

[17] D. J. McFarland, L. M. McCane, S. V. David, and J. R. Wolpaw. Spatial filter selection for EEG-based communication. Electroencephalography and Clinical Neurophysiology, 103(3):386 - 394, 1997.

[18] F. R. Najafabadi, A. Rahimi, P. Kanerva, and J. M. Rabaey. Hyperdimensional computing for text classification. Design, Automation Test in Europe Conference Exhibition (DATE), University Booth, 2016.

[19] K. Nishihara, N. Taya, and T. Kanoh. A consideration of realizing the brain inspired computer. In Proceedings of the 9th EAI International Conference on Bio-inspired Information and Communications Technologies (Formerly BIONETICS), BICT'15, pages 495-496, ICST, Brussels, Belgium, Belgium, 2016.

[20] A. Rahimi, S. Benatti, P. Kanerva, L. Benini, and J. M. Rabaey. Hyperdimensional biosignal processing: A case study for EMG-based hand gesture recognition. In IEEE International Conference on Rebooting Computing, October 2016.

[21] A. Rahimi, P. Kanerva, and J. M. Rabaey. A robust and energy efficient classifier using brain-inspired hyperdimensional computing. In Low Power Electronics and Design (ISLPED), 2016 IEEE/ACM International Symposium on, August 2016.

[22] M. Sahlgren. An introduction to random indexing. In Methods and Applications of Semantic Indexing Workshop at the 7th International Conference on Terminology and Knowledge Engineering, TKE 2005, 2005.

[23] D. Widdows and T. Cohen. Reasoning with vectors: A continuous model for fast robust inference. Logic Journal of the IGPL, 23(2):141-173, 2014. 